Check for updates

Cite this: RSC Adv., 2019, 9, 3462

\title{
Effects of amphiphilic monomers and their hydrophilic spacers on polyacrylamide hydrogels
}

Received 23rd November 2018 Accepted 11th January 2019

DOI: $10.1039 / c 8 r a 09644 a$

rsc.li/rsc-advances

\author{
Yudong Liu, Zhiying Li, Jianan Xu, (D) Bao Wang, Fengya Liu, Ruiqi Na, \\ Shaowei Guan (D) and Fengqi Liu*
}

Hydrogels based on physical interactions have been extensively studied due to their special network structure and excellent mechanical properties. In this paper, a series of hydrogels based on hydrophobic interactions were prepared via the free-radical copolymerization of acrylamide and polymerizable amphiphilic monomers dodecanol polyoxyethylene $(n)$ acrylates (AEO- $n-A C, n=3,7,9,15,23$ ) by a simple and facile method. The prepared single-network hydrogels cross-linked by the self-assemble AEO- $n$-AC micelles acting as cross-linkers exhibited great tensile strength of $0.45 \mathrm{MPa}$ and excellent compression strength of 4.5 $\mathrm{MPa}$. Transmission electron microscopy tests reflected that the morphologies of the self-associated micelles were determined by the hydrophilic segment of the amphiphilic monomers, which further affected the mechanical properties of the hydrogel. Amphiphilic monomer with appropriate length of hydrophilic spacers could significantly enhance the tensile strength of the hydrogel. Meanwhile, amphiphilic monomers with long hydrophilic segment were advantageous for the compression properties of the hydrogel. Furthermore, the hydrogels exhibited excellent micro self-repair ability during the cycling tensile and loading-unloading test even at the strain and compression were 400\%, 0.95, respectively. This discover of hydrophilic spacer effect is of great significance for the design of physical interaction-based hydrogels with high strength and compression properties.

\section{Introduction}

Hydrogels are formed by the cross-linking of hydrophilic polymer chains in aqueous microenvironment. ${ }^{1}$ With unique threedimensional network structures and biocompatibilities, hydrogels are widely used in water-retaining, ${ }^{2}$ drug delivery, ${ }^{\mathbf{3}, \mathbf{4}}$ tissue engineering, ${ }^{5,6}$ and soft biomimetic machines. ${ }^{6}$ However, the applications of traditional hydrogels are limited by their poor mechanical properties, such as brittleness and low strength. The lack of an efficient energy dissipation mechanism in the gel network, as shown in Fig. 1(a), leads to the hydrogels exhibiting low resistance to crack extension.,

In the last few years, many strategies including double network hydrogels, ${ }^{9}$ microgel-reinforced hydrogels, ${ }^{10}$ nanocomposite hydrogels, ${ }^{11}$ supramolecular hydrogels ${ }^{\mathbf{1 2}}$ and hydrophobic association hydrogels ${ }^{\mathbf{1 3}}$ have been developed to enhance the strength of hydrogels. Among them, physical cross-link hydrogel, have attracted great interests, due to their unique reversible cross-linking structure and energy dissipation mechanism. Incorporation of hydrogen bonds, oppositely charged interactions and hydrophobic interactions are the most common and effective strategies for preparing physically

College of Chemistry, Jilin University, Changchun 130012, People's Republic of China. E-mail: guansw@jlu.edu.cn; liufengqi@jlu.edu.cn crosslinked hydrogels with excellent properties. ${ }^{\mathbf{1 4 - 1 6}}$ Inspired by the widespread presence of hydrophobic interactions in biological systems, ${ }^{\mathbf{1 7}}$ hydrophobic association hydrogels have been widely studied due to their facile prepared, excellent mechanical properties and self-healing ability. The hydrophobic interaction between the hydrophobic chains act as reversible crosslinking point, which could efficiently dissipate the crack energy. ${ }^{18}$ As illustrated in Fig. 1(b), the reversible disengagements of the hydrophobes from the hydrophobic associations can effective dissipate the crack energy.
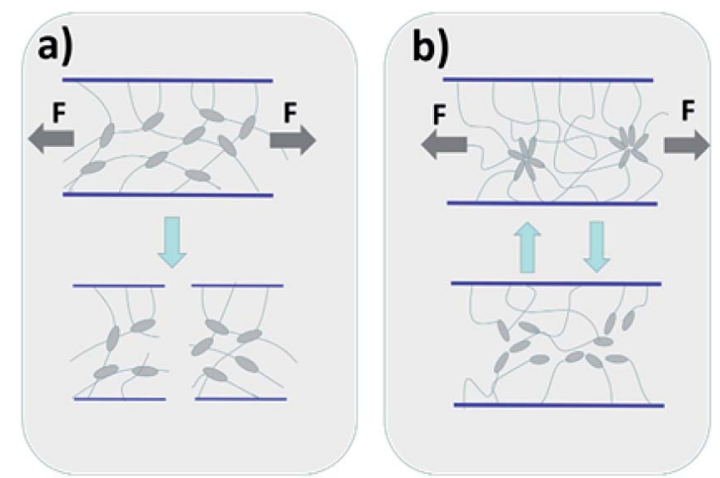

Fig. 1 Diagram of gel network crack propagation of chemicallycrosslinked hydrogel (a) and hydrophobic association hydrogel (b). 
Recently, the designing of hydrophobic monomers with new chemical structure to fabricate hydrophobic interaction-based hydrogel has made great progress. ${ }^{19-21}$ For example, Arindam Banerjee report a series of amphiphilic tyrosine based selfhealable metallo-hydrogels and one single amino acid (phenylalanine) based pyrene conjugated low molecular weight hydrogelator. ${ }^{22,23}$ The stiffness and self-healing property of the amphiphilic tyrosine-based hydrogels can be nicely tuned by varying the alkyl chain length of these amphiphiles and the phenylalanine (Phe) derivative-based hydrogel shown well super-gelation efficiency and thixotropic property. Okay reported a series of hydrogels prepared by incorporating of $n$ alkylacrylamides or $n$-alkyl methacrylates with an alkyl chains length between 4 and 12 carbon atoms into PAAM network, ${ }^{18,24}$ the low water solubility of long-chain hydrophobes, surfactant (usually SDS) was frequently used to grow the micelles. ${ }^{25,26}$ The hydrogel exhibited different toughness and strength with different alkyl chain length. The hydrogel with longer alkyl chains exhibited higher strength and toughness and revealed the relationship between the alkyl chain of hydrophobe and the properties of the gel strength, toughness and elongation. However, the strength and elongation of the prepared gels by this way were just $50 \mathrm{kPa}$ and $200 \%$, respectively, duo to their shorter alkyl chain, which was insufficient for their application. Subsequently, they investigated the effect of longer alkyl on gels properties. ${ }^{27}$ The hydrophobes with longer alkyl chains such as stearyl methacrylate (C18) or dococyl acrylate (C22) was copolymerized into networks. While, due to the very low water solubility of the C18 and C22, they could only be dissolved in a mixed solution of SDS and $\mathrm{NaCl}$. It was of interest to us that the strength of the synthesized gel after swelling (extraction of SDS) was much higher than the original prepared gel. The author held the opinion that SDS micelle result in the associations weak and reversible owing to the solubilization of the hydrophobic moieties. When there was no SDS present (after extraction), the associations would become strong and the gel behaved like being covalently crosslinked. Inspired by this, we wondered whether the hydrophobic association gel without SDS would have a better performance if it could be synthesized directly.

In our previous reports, we fabricated a series of hydrophobic association hydrogels with good properties by incorporating of modified octylphenol polyoxyethylene ether acrylate (OP- $n$-AC) as hydrophobic monomers into PAAM network. ${ }^{28-30}$ The strong hydrophobic benzene ring-like structure on the OP$n$-AC enhanced the hydrophobic interaction between the hydrophobic monomers and brought about higher mechanical strength. However, these monomers could only be dissolved in the SDS micelles.

In recent studies, we fabricated HA-gel based on modified fatty alcohol polyoxyethylene ( $n$ ) ether acrylate (AEO-AC- $m-n$ ) as hydrophobic monomer and Acrylamide as hydrophilic monomer. ${ }^{31-33}$ The effect of the structure of the alkyl chain and the synergistic effect of two hydrophobic monomers on the mechanical properties of the hydrogel were investigated. In the present work, instead of focusing on the hydrophobic segment, we focus on the other end of the hydrophobic monomers: the "hydrophilic segment". We chose a series of AEO- $n$-ACs as amphiphilic monomers. The hydrophobic segment was an alkyl chain containing 12 carbon atoms, which provides sufficient hydrophobic interaction. The hydrophilic segment was EO chain with different lengths $(n)$ to choose, which endowed the monomer favorable hydrophilicity and avoid SDS added. And in order to understand the effect of hydrophilic spacers (EO) on the gel mechanical properties, the mechanism of hydrophilic spacers on gel properties was investigated for the first time by TEM, SEM, tensile test and compression experiments. Moreover, unlike previous reports, these prepared gels can exhibit self-healing ability without the presence of SDS surfactant.

\section{Results and discussion}

FT-IR spectral analysis was employed to confirm the introduction of amphiphilic monomer AEO- $n$-AC and the results were shown in Fig. 3. In view of the spectra of AEOs were almost the same, the spectral of AEO-3 was selected as a representative. In the spectrum of AEO-3, distinctive adsorption broad peak appeared at $3470 \mathrm{~cm}^{-1}$, corresponding to the stretching vibrations of $-\mathrm{OH}$. In contrast, the above-mentioned peak disappears in the IR spectrum of AEO- $n$-AC. Besides, the position of the absorption peak at $1735 \mathrm{~cm}^{-1}$ corresponded to stretching vibration of $\mathrm{C}=\mathrm{O}$; the peak at $1639 \mathrm{~cm}^{-1}$ corresponded to the stretching vibrations of $\mathrm{C}=\mathrm{C}$; the peak at $1405 \mathrm{~cm}^{-1}$ corresponded to the shear vibrations of $=\mathrm{CH}_{2}$ and the peak at $1195 \mathrm{~cm}^{-1}$ corresponded to the asymmetric stretching vibration of $\mathrm{C}-\mathrm{O}-\mathrm{C}$. These were quite different from curve AEO- $n$.

${ }^{1} \mathrm{H}$-NMR spectra of AEO- $n$ and AEO- $n$-AC were shown in Fig. 4. For the spectrums of AEO- $n$ were almost the same, the spectrum of AEO-3 was shown as a representative. Intense resonance peaks appeared at 5.85, 6.14 and 6.43 ppm (Fig. 4(b, a and c), respectively) in the AEO- $n$-AC ${ }^{1} \mathrm{H}-\mathrm{NMR}$ spectrum, which were assigned to the protons of $-\mathrm{CH}=\mathrm{CH}_{2}$. The peak at $4.32 \mathrm{ppm}$ (Fig. 4(d)) corresponded to the methylene protons which links to ester group. While, the peak at $2.43 \mathrm{ppm}$

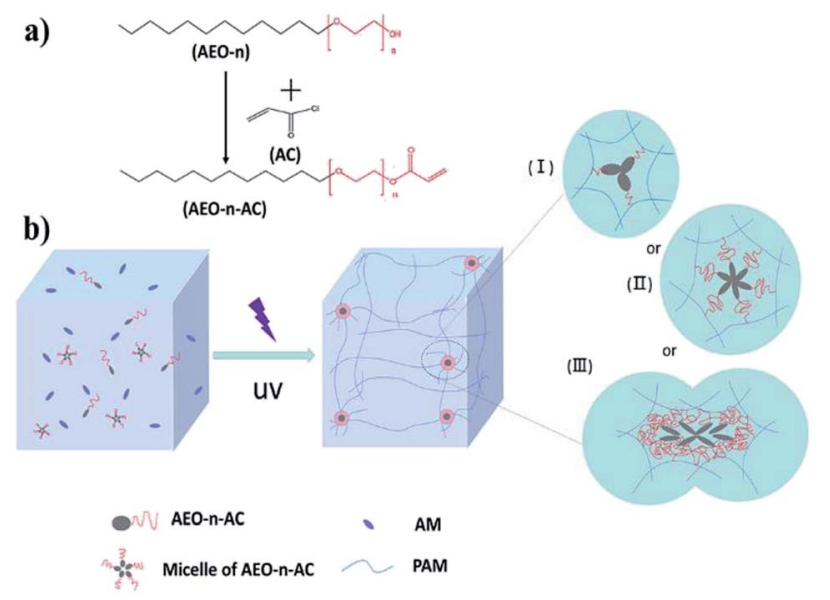

Fig. 2 (a) Schematic illustration for the synthesis and structure of $A E O-n-A C$ and (b) the synthesis of (I) shorter-EO $\mathrm{AEO}_{3}$-gel, (II) long$\mathrm{EO} \mathrm{AEO}_{7}$-gel, or $\mathrm{AEO}_{9}$-gel (III) longer-EO $\mathrm{AEO}_{15}$-gel or $\mathrm{AEO}_{23}$-gel. 


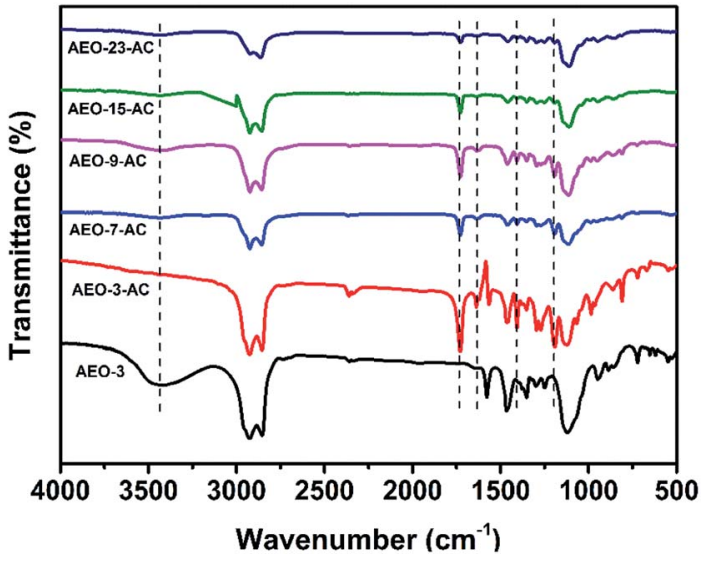

Fig. 3 FTIR spectra of AEO- $n$ and AEO- $n-A C$.

disappeared which belongs to the hydroxyl proton in the AEO-3 spectrum. The IR and ${ }^{1} \mathrm{H}-\mathrm{NRM}$ spectra illustrated that AEO- $n-\mathrm{AC}$ was successfully synthesized.

The morphologies of these AEO- $n$-AC micelles were investigated by means of TEM. Fig. 5 shown the different configuration of AEO- $n$-AC micelles. As shown in Fig. 5(a), the micelles formed by short hydrophilic EO-chain AEO-3-AC were homogeneous and their diameter were about $1 \mu \mathrm{m}$, usually existed in the form of single nucleus. The diameter of the micelles associated by AEO-7-AC and AEO-9-AC were about $1.5 \mu \mathrm{m}$. The morphologies of the micelles became relatively larger and their structure were relatively uniform. While, with longer hydrophilic spacers, morphologies of AEO-15-AC and AEO-23-AC micelles become more complex. The diameters of the micelles were about $2 \mu \mathrm{m}$ and their structure changed from single-nucleus to multinucleus. As shown in the Figure 5(a-e), the micelles formed by AEO-3-AC was a small ball. AEO-7-AC and AEO-9-AC micelles

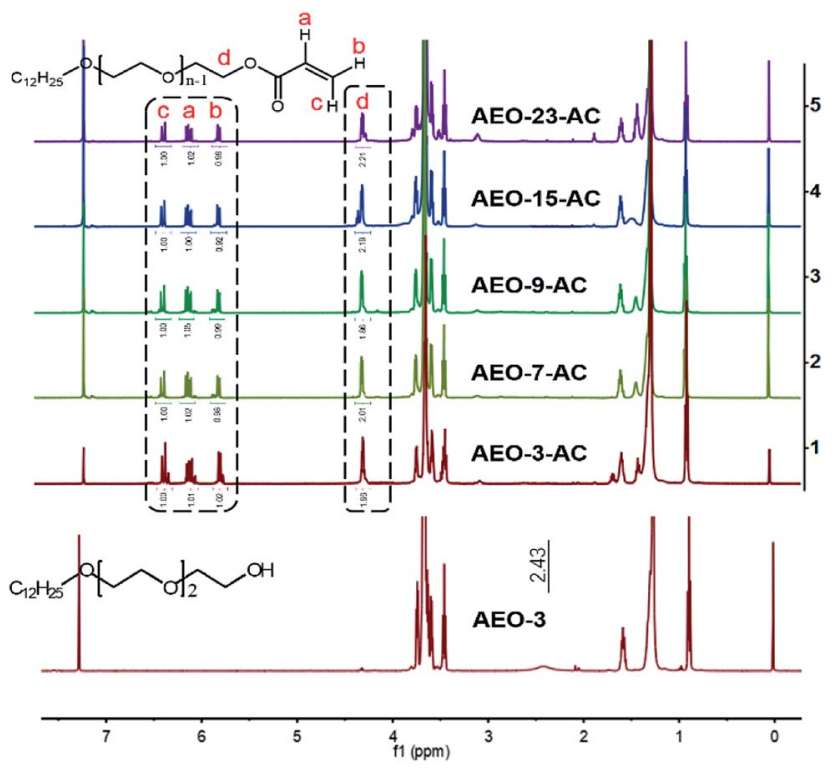

Fig. $4{ }^{1} \mathrm{H}-\mathrm{NMR}$ spectra of AEO- $n$ and AEO- $n-\mathrm{AC}$.

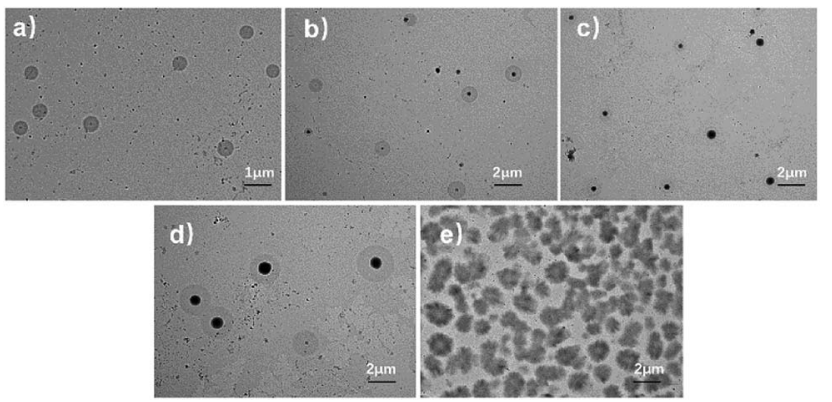

Fig. 5 Microscopic morphology of AEO-n-AC micelle. (a-e) AEO-3AC, AEO-7-AC, AEO-9-AC, AEO-15-AC, AEO-23-AC.

were also spherical but much bigger. While, the micelles formed by AEO-15-AC and AEO-23-AC tend to collision and coalescence became dumbbell-shaped owing to its ultralong hydrophilic spacers.

The morphologies of these $\mathrm{AEO}_{n}$-gels were obtained by freeze-drying method, which were reflected in the SEM diagram. Fig. 6 shows that a three-dimensional network was formed in those gels and a lot of cellular structures appeared. Interesting, the cellular structure in $\mathrm{AEO}_{3}$-gel (Fig. 6(a)) was small, and the diameter was less than $10 \mu \mathrm{m}$, but the pore wall was thick. With the length of hydrophilic EO chain $(n)$ increased, the cellular structure of the prepared HA-gel become more uniform and arrange regularly, as Fig. 6(b) and (c) shown, their diameters were about $20 \mu \mathrm{m}$. However, when it comes to $\mathrm{AEO}_{15}$-gel and $\mathrm{AEO}_{23}$-gel, the longer hydrophilic spacers in micelles make the association of hydrogels less compact, resulting in the flake structures (Fig. 6(d) and (e)). Despite the SEM test could not reflect the actual structure of hydrogel, the result indicated that the hydrophilic spacers of the amphiphilic monomers have a crucial effect on the microstructure of hydrogel.

In order to illustrate the effect of hydrophilic segment length on the mechanical properties of hydrogel, we prepared a series of hydrogels with AEO- $n$-AC/AM of $2 \%$ for comparison. As shown in Fig. 7, the tensile strength was only $0.12 \mathrm{MPa}$ for short$\mathrm{EO} \mathrm{AEO}_{3}$-gel and $0.17 \mathrm{MPa}, 0.18 \mathrm{MPa}$ for long-EO $\mathrm{AEO}_{15}$-gel, $\mathrm{AEO}_{23}$-gel. And, their toughness was less than $0.5 \mathrm{MJ} \mathrm{m}^{-3}$. In contrast, the strengths of $\mathrm{AEO}_{7}$-gel and $\mathrm{AEO}_{9}$-gel with moderate

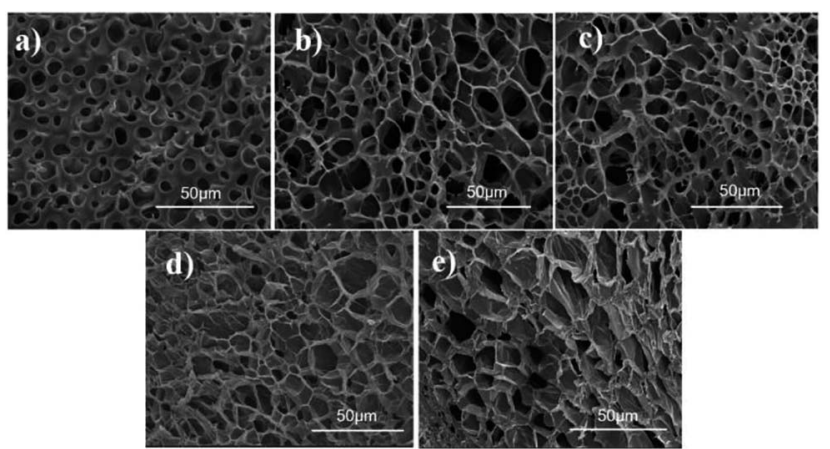

Fig. 6 SEM images of $\mathrm{AEO}_{n}$-gels: (a) $\mathrm{AEO}_{3}$-gel, (b) $\mathrm{AEO}_{7}$-gel, (c) $\mathrm{AEO}_{9}$ gel, (d) $\mathrm{AEO}_{15}$-gel, (e) $\mathrm{AEO}_{23}$-gel. 
(a)

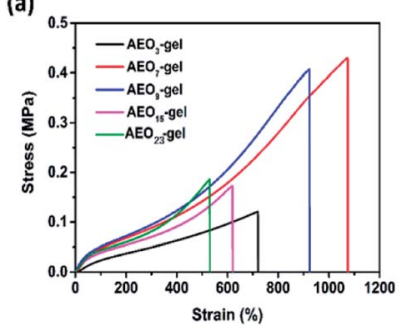

(b)

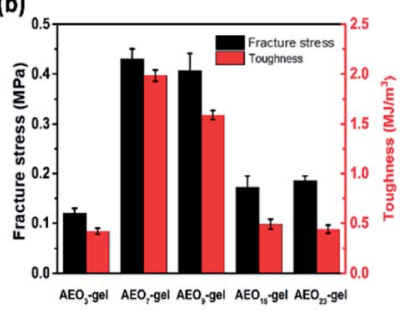

Fig. 7 (a) Tensile stress-strain curves of hydrogels with different AEO$n-A C$ and (b) stress and toughness of these hydrogels.

hydrophilic segment (EO) length were $0.45 \mathrm{MPa}$ and $0.42 \mathrm{MPa}$ and their toughness was 2.0 and $1.6 \mathrm{MJ} \mathrm{m}^{-3}$, respectively. Furthermore, Fig. 6(a) also reflected that the gels prepared by AEO-7-AC and AEO-9-AC have better elongation at break.

Our previous studies have shown that the strength of hydrogel was related to the strength of association microregion and crosslink density. ${ }^{34}$ As explained in Fig. 2(b), the association microregion acts as crosslinking point in hydrophobic association hydrogels. For $\mathrm{AEO}_{3}$-gel, the hydrophilic spacers were too short to engender enough solubilization. The short hydrophilicchain monomer results in the formation of associated microregion containing little hydrophobic spacers, as shown in Fig. 2(b-I). As a result, many weak cross-linking points made the hydrogel weak strength and toughness. In other way, the ultralong hydrophilic EO chains of AEO-15-AC and AEO-23-AC hindered the formation of stable micelles. As Fig. 2(b-III) shown, Association microregion of $\mathrm{AEO}_{15}$-gel and $\mathrm{AEO}_{23}$-gel were more prone to collision and coalescence, resulting in a low strength of the prepared gel. While, micelles formed by AEO-7$\mathrm{AC}$ and $\mathrm{AEO}-9-\mathrm{AC}$ with moderate hydrophilic spacers length were stable and larger spherical, so their association microregion were regular and compact, as shown in Fig. 2(b-II). And the prepared gel exhibits better mechanical properties. Therefore, the results proved that the length of a suitable hydrophilic segment and the formation of stable micelle are necessary for preparing high strength and toughness hydrogel.

In modern biomedical field, many artificial materials are required to have excellent deformation resilience and selfrecovery property. Since, some soft tissues, such as muscles and ligaments, have to undergo multiple deformations over a long period time. So, the excellent deformation resilience was essential for the practical application of hydrogels. In order to obtain the performance of the hydrogels under continuous external force. We adopted two methods to discuss the resilience of these hydrogels in different ways. One was continuous stretching, and there was no waiting time between each cycle, the result was shown in Fig. 8-1. The second way was intermittent stretching, with a 3 min interval between each cycle, the result was shown in Fig. 8-2. The results showed that these gels had good recovery capabilities, except for slight strength reduction due to weak and ineffective cross-linking point failure during the first cycle.

As shown in Fig. 8-1 during continuous stretching, the strength of the gel decreased after each cycle, indicating that the

1)

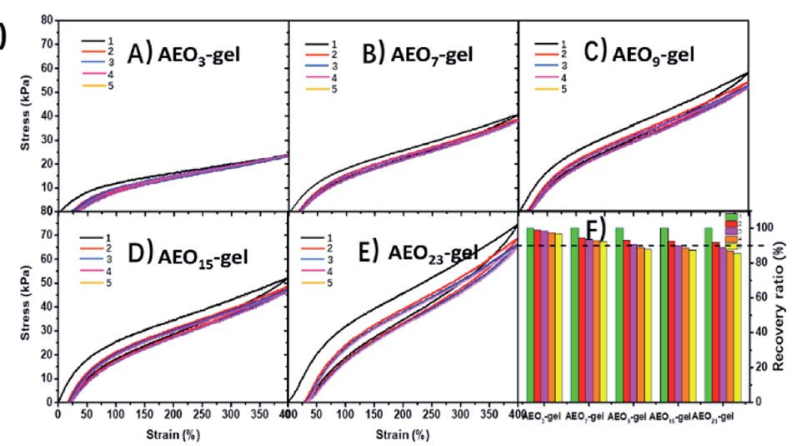

2)

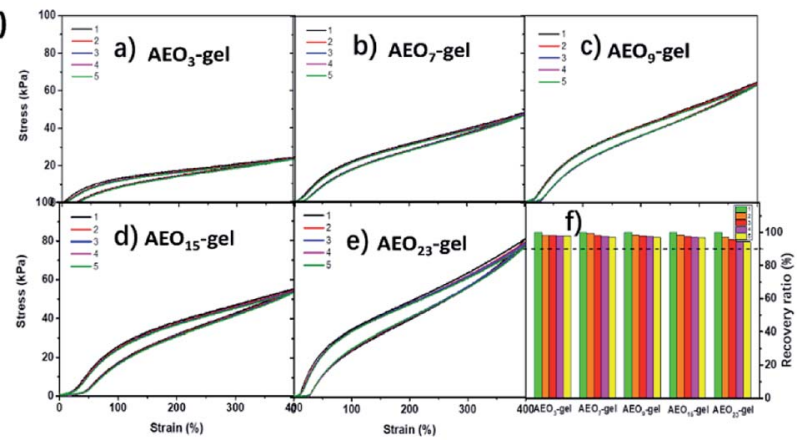

Fig. 8 Cyclic stress-strain curves of $\mathrm{AEO}_{n}$-gels at the strain of $400 \%$ (1) without waiting time and (2) with 3 min waiting time. (F) and (f) the corresponding recovery ratio.

association microdomains were destroyed. It was worth noting that the strength of gels with longer EO chains decreased more rapidly (Fig. 8-1(f), from $100 \%$ to $85 \%$ for $\mathrm{AEO}_{23}$-gel), indicating that the microdomains of the gel with longer EO chains were more susceptible to damage at the same strain. As a contrast, in Fig. 8-2 after a short waiting time, the stress-strain curves of all gels could almost coincide (recovery rate is more than 95\%), which proved that these gels have good micro-self-repairing ability, i.e., hydrophobic association micro-domains that were broken could be associated again. This indicates that the molecular chain is not broken during the stretching process, but only the destruction and deformation of the physical association microdomain. Moreover, hydrogels with shorter hydrophilic spacers have better microscopic self-healing capabilities. It was possible that the long hydrophilic spacers which acts as the spacer hindered the reassociation of the hydrophobic spacers after the deformation of the associating microregion, leading to the lower self-recovery efficiency. Compared with the hydrogels based on hydrophobic interactions and metalligand, ${ }^{17}$ these hydrogels prepared by amphiphilic monomers AEO- $n$-AC had superior deformation resilience.

Besides the tensile tests, compression tests were also performed to evaluate the compression-resistant property of the hydrogels. Fig. 9-1 shows the compression loading-unloading curves and the corresponding dissipated energy of hydrogels. There were no compression fractures were observed for all these hydrogels even at the compression ratio as high as 0.95 . The corresponding compressive strengths were $1.8 \mathrm{MPa}$, 3.5 MPa,

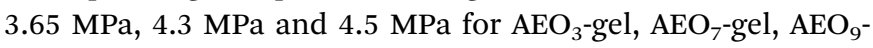
gel, $\mathrm{AEO}_{15}$-gel and $\mathrm{AEO}_{23}$-gel for the first loading-unloading test. 

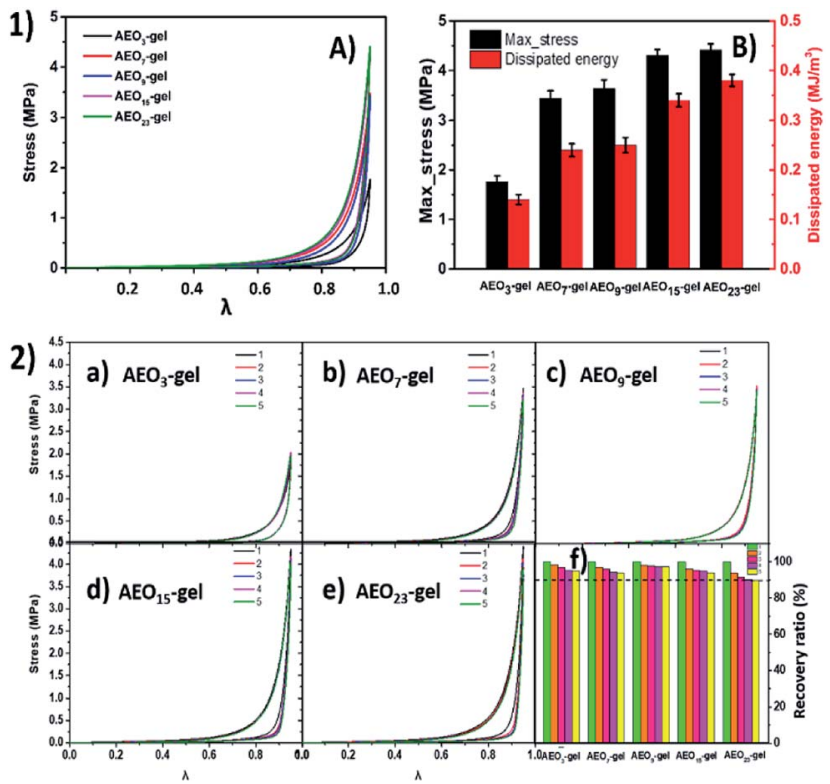

Fig. 9 (1-A) Compression loading-unloading curves at 0.95 compression ratio $\lambda$ for $\mathrm{AEO}_{n}$-gels. (1-B) The corresponding stress and dissipated energy calculated form (A). (2-a-e) Cyclic compression loading-unloading curves for $\mathrm{AEO}_{n}$-gels. (2-f) the corresponding recovery ratio for (a-e).

And the dissipated energy also increased from $0.13 \mathrm{MJ} \mathrm{m}^{-3}$ to $0.39 \mathrm{MJ} \mathrm{m}^{-3}$. It was obviously that, unlike the tensile test, compressive strength of the gels exhibits a correlation with the hydrophilic spacer. Hydrogel cross-linked with longer hydrophilic spacers monomer have higher compressive stress and more energy dissipation which were stronger than most other single-network hydrogel, indicating excellent compressionresistant property.

As Fig. 9-2 shows, five-run cyclic compression tests were performed to discuss the effect of different spacers on compression-recovery ability of these hydrogel. Obviously, after five cycles of compression, the gel with a longer spacer has a lower intensity recovery rate. We hypothesize that a large amount of hydrophilic EO spacers around the hydrophobic segment will significantly make the micelles larger, as the Fig. 2(b-III) or 4(e) shows. Thus, on one hand, the gel formed by the large microdomains will exhibit a higher resistance to deformation when compressed. However, on the other hand, the recover ability of the microdomain consist of more softsegment EO chains will become weaker.

Self-healing is critical for the application of hydrogels. Fig. 10 was the strain-stress curves of the self-healing hydrogels. Since self-healing was assisted by heat, we named it thermal healing. The inset in Fig. 10 showed the thermal healing progress of the hydrogels. The gel rod was cut into two parts, one of which was dyed blue by methylene blue. After that, the two parts were wrapped closely by PVC film and then the two parts were totally adhered after $24 \mathrm{~h}$ in $50{ }^{\circ} \mathrm{C}$. It was shown that the stress and strain of self-healing $\mathrm{AEO}_{3}$-gel were $20 \mathrm{kPa}, 75 \%$, respectively. While the strength and stress of thermal-healing $\mathrm{AEO}_{23^{-}}$ gel could reach $80 \mathrm{kPa}, 190 \%$, respectively. The hydrogel with

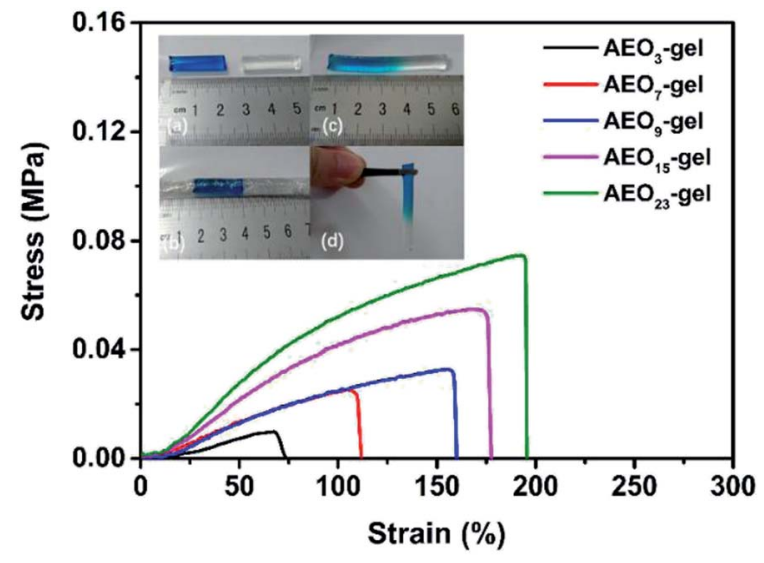

Fig. 10 Tensile stress-strain curves of the thermal healing $\mathrm{AEO}_{n}$-gels and the photographs of thermal healing process.

longer EO space exhibited higher tensile strength and extensibility after thermal healing.

It is worth noting that this macro thermal healing phenomenon was different from the previous micro selfrecovery. Previous tensile and compression experiments have shown that hydrogels with long hydrophilic segment had weaker microscopic self-recovery ability than that of short hydrophilic segment. However, macroscopic thermal healing experiments showed the opposite result. We considered that the association microregions of the hydrogels were not completely destroyed in the tensile and compression tests, and the hydrophobic spacers were still concentrated near the associative microregions. Self-recovery mainly depended on the chain motion of hydrophobic spacers. However, in the thermalhealing experiment, the association microregions on the fracture surface were completely broken. The reformation of association microregions depends on the motion of the whole molecular chain. The molecules with long hydrophilic spacers have better chain movement ability, which will drive the association of hydrophobic spacers on different main chain, thus, shows better macroscopic self-healing ability.

\section{Materials}

Linear AEO (AEO- $n, n=3,7,9,15,23$; Chemical pure) were purchased from Ningbo Lejia Chemical Co. (china). Acryloyl chloride (AC, 98\%) was provided by Energy Chemical Co. (China). Acrylamide (AM, 98.0\%), triethylamine (TEA; analytical reagent) and tetrahydrofuran (THF; analytical reagent) were purchased from Tianjin Guangfu Fine Chemical Research Institute (China). The photoinitiator $\alpha$-ketoglutaric acid ( $\alpha$-ka, 99\%) was purchased from Aldrich. TEA and THF were predistillation before used. Other reagents were used without further purification.

\section{The synthesis of AEO- $n-A C$}

Polymerizable amphiphilic monomers AEO- $n$-ACs were synthesized by the reaction of acryloyl chloride with the hydroxyl groups of AEO- $n$, which have been described detailed in our 
previous report. ${ }^{29}$ Briefly, AEO- $n$ (0.05 mol) and TEA (0.075 mol) was dissolved in $50 \mathrm{ml}$ THF solvent. AC (0.1 mol) diluted in $10 \mathrm{ml}$ THF was added drop-wise in $30 \mathrm{~min}$ after the solution was cooled to $0{ }^{\circ} \mathrm{C}$. The mixture was stirred at $0{ }^{\circ} \mathrm{C}$ for $5 \mathrm{~h}$ and $24 \mathrm{~h}$ at room temperature. After removing the precipitated hydrochloride salt, the AEO- $n$-AC was dried with a rotary evaporator under vacuum at room temperature. The yields of AEO- $n$-ACs were among $90-94 \%$.

\section{Preparation of the hydrogels}

The hydrogels were prepared by free-radical micellar copolymerization as shown in Fig. 2(b). As a typical example, $50 \mathrm{mmol}$ AM $(3.55 \mathrm{~g})$ and $1 \mathrm{mmol}$ AEO- $n$-AC $(0.372 \mathrm{~g}$ AEO-3-AC or $0.548 \mathrm{~g}$ AEO-7-AC, 0.636 g AEO-9-AC, 0.900 g AEO-15-AC, 1.252 g AEO23-AC) were dissolved in $14.20 \mathrm{ml}$ distilled water. After the solution was stirred for $8 \mathrm{~h}$, the $\alpha$-ka ( $40 \mathrm{mg}$ ) was added into the mixture and stirred for another 30 min under nitrogen atmosphere. Then, the mixed solutions were transferred into a test tube (containing five $6 \mathrm{~mm}$ o.d. $3.50 \mathrm{~mm}$ length plastic pipes) and sealed. Finally, the test tube was irradiated by $365 \mathrm{~nm}$ UV light for $5 \mathrm{~h}$. The prepared hydrogel was named $\mathrm{AEO}_{n}$-gel. The number $n$ indicates that the hydrogel was synthesized using AEO- $n$-AC amphiphilic monomer. For all the samples, the concentration of AM and $\alpha$-ka were fixed at $20 \mathrm{wt} \%$ and $0.2 \mathrm{wt} \%$, respectively. All the other five kinds of hydrogels were prepared in the same way.

\section{Characterization}

The Fourier transform infrared (FTIR) spectra of AEO- $n$-AC were recorded on a Nicolet 360 FEIR spectrophotometer. The samples were cast on $\mathrm{KBr}$ plates for characterization. All 500 MHz NMR studies were carried out on a Bruker $500 \mathrm{MHz}$ spectrometer at $298 \mathrm{~K}$ in $\mathrm{CDCl}_{3}$ maintaining the concentration 4-10 mM. The morphologies of the different AEO- $n$-AC micelles were observed by transmission electron microscopy (TEM). TEM samples were prepared by the drying of a drop of a dilute aqueous solution of AEO- $n$-AC micelle onto a carbon-coated copper grid. TEM analysis was performed on a JEM-2100F TEM instrument operating at $200 \mathrm{kV}$ in bright-field mode. The microstructure of these hydrogels in the freeze-dried sample were observed by scanning electron microscopy (SEM; Nova NanoSEM 450) with an accelerating voltage of $10 \mathrm{kV}$. In order to keep the structure of the hydrogels unchanged after freezing, these prepared gels were quickly frozen by liquid nitrogen for several minutes. Then, the freeze-dried samples were obtained under a vacuum of $0.1 \mathrm{~Pa}$ for $24 \mathrm{~h}$ to thoroughly remove the water. Before SEM observation, the surface was coated with a thin layer of gold by the sputtering method.

\section{Measurement of the mechanical properties of the hydrogels}

The tensile tests and compression test were carried out on a commercial Universal testing machine AG-I (Shimadzu Corp.,
Kyoto, Japan) with a $1 \mathrm{kN}$ load cell. For tensile test, the samples were prepared by the method described previously at a stretch velocity $100 \mathrm{~mm} \mathrm{~min}^{-1}$. For compression test, the hydrogel specimens (cylinder, $21 \mathrm{~mm}$ in diameter and $10 \mathrm{~mm}$ in height) was measured with a crosshead speed of $5 \mathrm{~mm} \mathrm{~min}^{-1}$. Moreover, the cycling tensile and compression tests were also performed to characterize the self-recovery behavior of the prepared hydrogels at the speed of $20 \mathrm{~mm} \mathrm{~min}^{-1}, 5 \mathrm{~mm} \mathrm{~min}^{-1}$, respectively. All these mechanical tests were carried out at room temperature and each mechanical test was performed 5 times. The self-healing process and re-tensile test of the prepared hydrogels were recorded using the images obtained with an ordinary camera.

\section{Conclusion}

Hydrogels based on hydrophobic interaction were synthesized by simple copolymerization of a series of amphiphilic monomers and acrylamide. For the first time, we systematically investigated the effects of hydrophilic spacers of amphiphilic monomers on the mechanical properties of hydrogels. It was discovered that the length of hydrophilic spacers had a significant effect on the microstructure and performance of the hydrogels. Hydrogel with appropriate length hydrophilic spacers, such as 7 or 9 EO hydrophilic chains, showed better tensile strength and toughness. And longer hydrophilic spacers could improve the compression performance of the hydrogel. TEM and SEM experiments also revealed the effect of hydrophilic spacers on the morphology of the self-associating micelles and the microstructure of the synthesized hydrogel. Cyclic tensile and cyclic compression tests have proved that these hydrogels have an efficient energy dissipation mechanism and great self-recovery ability. Moreover, we discovered that hydrophilic spacers have diverse effects on micro self-recovery and macro thermal-healing of hydrogels. Shorter hydrophilic chain was conducive to microscopic self-recovery, while the longer hydrophilic chain was more conducive to macroscopic thermal healing. This study enriches the study of hydrogels based on hydrophobic interactions from a new perspective. It provides a new idea for the development and design of hydrogels based on physical interactions.

\section{Conflicts of interest}

There are no conflicts to declare.

\section{Acknowledgements}

This research was supported by a grant from the National Natural Science Foundation of China (contract grant number 21174053).

\section{Notes and references}

1 Y. S. Zhang and A. Khademhosseini, Science, 2017, 356, 3627-3637. 
2 X. W. Peng, L. X. Zhong, J. L. Ren and R. C. Sun, J. Agric. Food Chem., 2012, 60, 3909-3916.

3 R. F. Donnelly, T. R. Singh, M. J. Garland, K. Migalska, R. Majithiya, C. M. McCrudden, P. L. Kole, T. M. Mahmood, H. O. McCarthy and A. D. Woolfson, Adv. Funct. Mater., 2012, 22, 4879-4890.

4 G. Erdodi and J. P. Kennedy, Prog. Polym. Sci., 2006, 31, 1-18. 5 H. J. Kong, M. K. Smith and D. J. Mooney, Biomaterials, 2003, 24, 4023-4029.

6 E. Eisenbarth, Adv. Eng. Mater., 2010, 9, 1051-1060.

7 R. E. Webber, C. Creton, H. R. Brown and J. P. Gong, Macromolecules, 2007, 40, 2919-2927.

8 K. Haraguchi, K. Uyama and H. Tanimoto, Macromol. Rapid Commun., 2011, 32, 1253-1260.

9 J. P. Gong, Y. Katsuyama, T. Kurokawa and Y. Osada, Adv. Mater., 2003, 15, 1155-1158.

10 J. Hu, K. Hiwatashi, T. Kurokawa, S. M. Liang, Z. L. Wu and J. P. Gong, Macromolecules, 2011, 44, 7775-7781.

11 N. Samadi, M. Sabzi and M. Babaahmadi, Int. J. Biol. Macromol., 2018, 107, 2291-2297.

12 X. Y. Dai, Y. Y. Zhang, L. N. Gao, T. Bai, W. Wang, Y. L. Cui and W. G. Liu, Adv. Mater., 2015, 27, 3566-3571.

13 D. C. Tuncaboylu, A. Argun, M. Sahin, M. Sari and O. Okay, Polymer, 2012, 53, 5513-5522.

14 H. Y. Lee, C. H. Hwang, H. E. Kim and S. H. Jeong, Carbohydr. Polym., 2018, 186, 290-298.

15 T. L. Sun, T. Kurokawa, S. Kuroda, A. B. Ihsan, T. Akasaki, K. Sato, M. A. Haque, T. Nakajima and J. P. Gong, Nat. Mater., 2013, 12, 932-937.

16 C. Bilici, V. Can, U. Nochel, M. Behl, A. Lendlein and O. Okay, Macromolecules, 2016, 49, 7442-7449.

17 Y. Deng, I. Hussain, M. M. Kang, K. W. Li, F. Yao, S. L. Liu and G. D. Fu, Chem. Eng. J., 2018, 353, 900-910.
18 S. Abdurrahmanoglu, V. Can and O. Okay, Polymer, 2009, 50, 5449-5455.

19 A. Argun, U. Gulyuz and O. Okay, Macromolecules, 2018, 51, 2437-2446.

20 B. Kurt, U. Gulyuz, D. D. Demir and O. Okay, Eur. Polym. J., 2016, 81, 12-23.

21 Q. Su, L. J. Duan, M. F. Zou, X. T. Chen and G. H. Gao, Mater. Chem. Phys., 2017, 193, 57-62.

22 S. Basak, J. Nanda and A. Banerjee, Chem. Commun., 2014, 50, 2356-2359.

23 J. Nanda, A. Biswas and A. Banerjee, Soft Matter, 2013, 9, 4198-4208.

24 S. Abdurrahmanoglu, M. Cilingir and O. Okay, Polymer, 2011, 52, 694-699.

25 X. Shi, W. Wang and A. Wang, Carbohydr. Polym., 2013, 94, 449-455.

26 C. Wang, C. G. Wiener, Z. W. Cheng, B. D. Vogt and R. A. Weiss, Macromolecules, 2016, 49, 9228-9238.

27 D. C. Tuncaboylu, M. Sari, W. Oppermann and O. Okay, Macromolecules, 2011, 44, 4997-5005.

28 G. Q. Jiang, C. Liu, X. L. Liu, Q. R. Chen, G. H. Zhang, M. Yang and F. Q. Liu, Polymer, 2010, 51, 1507-1515.

29 M. Yang, C. Liu, Z. Y. Li, G. Gao and F. Q. Liu, Macromolecules, 2010, 43, 10645-10651.

30 Z. Liang, T. T. Gao, J. N. Xu, Z. Y. Li, X. L. Liu and F. Q. Liu, Chem. Res. Chin. Univ., 2015, 31, 633-639.

31 Y. D. Liu, Z. Y. Li, N. Niu, J. Y. Zou and F. Q. Liu, J. Appl. Polym. Sci., 2018, 135, 46400-46407.

32 T. T. Gao, N. Niu, Y. D. Liu, X. L. Liu, G. Gao and F. Q. Liu, $R S C$ Adv., 2016, 6, 43463-43469.

33 X. Lv, S. L. Sun, H. D. Yang, G. Gao and F. Q. Liu, J. Appl. Polym. Sci., 2017, 134, 45196-45204.

34 G. Jiang, C. Liu, X. Liu, Q. Chen, G. Zhang, M. Yang and F. Liu, Polymer, 2010, 51, 1507-1515. 